157

THE POPULATION-LEVEL IMPACT OF EXERCISE TRAINING PROGRAMS TO PREVENT SPORTS INJURIES A CONTROLLED ECOLOGICAL EVALUATION BASED ON REDUCTIONS IN HOSPITAL-TREATED INJURIES

${ }^{1}$ Caroline F Finch, ${ }^{1}$ Muhammad Akram, ${ }^{1}$ Alex Donaldson, ${ }^{2}$ Belinda Gabbe, ${ }^{3}$ David Lloyd, ${ }^{1,4} \mathrm{Jill}$ Cook. ${ }^{1}$ Federation University Australia; ${ }^{2}$ Monash University Australia; ${ }^{3}$ Griffith University Australia; ${ }^{4}$ LaTrobe University Australia

\subsection{6/injuryprev-2016-042156.157}

Background To date, there have been very few attempts at setting broad-based sports injury prevention public health activity. A staged and evidence-informed approach towards developing and delivering a new lower limb injury prevention program (FootyFirst) for community-level Australian football. The National Guidance for Australian football Partnerships for Safety (NoGAPS) study is one of the first large-scale studies to use a controlled, ecological design to assess the effectiveness of a sports injury prevention intervention at the population-level. The aim of this talk is to present the population-level evaluation of FootyFirst

Methods FootyFirst was implemented during the 2012 and 2013 football seasons and its impact on injury rate evaluated through a controlled ecological study design applied to three distinct geographic regions (R1-3). Each region received a different combination of program + delivery mode: Region 1 (R1)-Full FootyFirst program + fully supported delivery in both 2012 and 2013; R2Full FootyFirst program + unsupported delivery (both 2012 and 2013); R3-No FootyFirst + no delivery in 2012 (control), full FootyFirst program + full delivery in 2013 . For each region, the numbers of hospital-treated (admissions and emergency department presentations) sports injury cases at the hospitals serving those regions were obtained from routine data collections. The in-football season monthly number of lower limb injuries (\#LLI) during 2006-2013 was modelled by an intervention time series. The sports injury data was "interrupted" at the beginning of 2012, to coincide with the starting of the FootyFirst delivery, and a second inter accounted for an administrative data change. The model was used to assess changes in slope of the trend lines preand post-intervention using a generalised least squares method.

Results There was a significant decline in \#LLI after the first delivery of FootyFirst only for R1 (pre-FootyFirst monthly increase of 0.15 cases; post-FootyFirst monthly decline of 2.62 cases; effect $p=0.005)$. The administrative data change led to an increase in the number of cases in all regions.

Conclusions After adjusting for seasonal effects, there was a significant reduction in \#LLI treated in hospitals in the region where FootyFirst was accompanied by full implementation support.

\section{PREVENTION OF ANKLE SPRAIN INJURIES IN YOUTH SOCCER AND BASKETBALL}

${ }^{1}$ Oluwatoyosi Owoeye, 'Luz Palacios-Derflingher, ${ }^{1,2}$ Carolyn Emery. 'Sport Injury Prevention Research Centre, Faculty of Kinesiology; ${ }^{2}$ Alberta Children's Hospital Research Institute, Faculty of Medicine, University of Calgary, Alberta, Canada

\subsection{6/injuryprev-2016-042156.158}

Background Sport participation is the leading cause of injury in youth and ankle sprain is the most common sport injury. This study evaluates the effectiveness of neuromuscular training (NMT) in reducing the risk of ankle sprains in youth soccer and basketball players. The secondary objective includes evaluation of sex, age, sport and previous lower extremity (LE) injury as independent risk factors for ankle sprain.

Methods A secondary data analysis of three cohort studies and two randomised controlled trials conducted in one season of play in youth soccer and basketball players (ages 11-18) in Alberta, Canada (2005-2011) was completed. The definition of ankle sprain and injury surveillance methodology was consistent in all studies. Multivariable Poisson regression analysis (controlling for clustering by team) was used to estimate incidence rate ratios (IRR) with 95\% confidence intervals (CI), considering confounding and effect modification (e.g., sex, age, sport, previous injury). Results There were 188 ankle sprains in 171 players in 2,265 study participants. Multivariable analysis suggests that NMT was protective for ankle sprain injury [IRR $=0.69$ (95\% CI: $0.45-$ 1.05)] based on the point estimate. Sex, age, sport and previous injury did not modify this effect. Independent risk factors for ankle sprain injury included previous LE injury history $[(\mathrm{IRR}=1.77$ (95\% CI: 1.34-2.35) $]$ and participation in basketball vs. soccer [(IRR $=1.98$ (95\% CI: $1.30-3.01)]$. The risk of ankle sprain injury did not differ by sex $[$ IRR $=1.01$ (95\% CI: 0.53-1.92)].

Conclusions Exposure to a NMT program is protective for ankle sprain injury in youth soccer and basketball players. Independent risk factors for ankle sprain in youth sport include basketball participation (vs. soccer) and previous LE injury. Future research should focus on optimising NMT programs for the prevention of ankle sprain injuries and maximising player-adherence to NMT programs in youth soccer and basketball.

\section{EMERGENCY MEDICAL SERVICES RESPONSE TIME AND PAEDIATRIC MORTALITY AND MORBIDITY IN THE URBAN SETTING}

${ }^{1}$ Amy B Couperthwaite, ${ }^{2,3}$ lan E Blanchard, ${ }^{4}$ Dirk A Chisholm, ${ }^{3,5}$ Christopher J Doig ${ }^{3,4}$ Alberto Nettel-Aguirre, ${ }^{2}$ Gregory Vogelaar, ${ }^{2}$ Wadhah Almansoori, ${ }^{4}$ Tania Embree, ${ }^{6,7}$ Don Voaklander, ${ }^{2,3}$ Brent E Hagel. 'Department of Kinesiology and Health Sciences, York University, Canada; ${ }^{2}$ Alberta Health Services Emergency Medical Services, Canada; ${ }^{3}$ Department of Community Health Sciences, University of Calgary, Canada; ${ }^{4}$ Department of Paediatrics, University of Calgary, Canada; ${ }^{5}$ Department of Critical Care Medicine and Internal Medicine, University of Calgary, Canada; ${ }^{6}$ Alberta Centre for Injury Control and Research, Canada; ${ }^{7}$ School of Public Health, University of Alberta, Canada

\subsection{6/injuryprev-2016-042156.159}

Background The standard response time benchmark for Emergency Medical Services (EMS) has been set at eight minutes or less for ground ambulances in many parts of the world. It has not been extensively studied, especially in paediatric patients who suffered a traumatic injury. As injury is the leading cause of death for those under the age of 18 it is important to determine if this benchmark for EMS response time may be a factor in paediatric mortality and morbidity outcomes.

Methods All paediatric calls made to EMS between April 2010 to September 2013 in the cities of Calgary and Edmonton, Alberta, Canada were examined to select patients who had suffered a traumatic injury. These records were then linked to emergency department records and hospitalisation records using a deterministic linkage strategy using personal healthcare number, sex, and receiving facility. Patients were excluded if they were $\geq 18$ years old, attended to outside of Calgary or Edmonton areas or suffered a medical complaint not related to an injury. Response time, the exposure, was defined as time of call to $9-1-1$ to arrival of ambulance on scene. Response time was dichotomized into $<8$ minutes and $\geq 8$ minutes. The main measure of morbidity was 
hospital admission. Adjusted risk ratios were calculated using Poisson regression.

Results 42620 patients were attended to between April 2010 and September 2013. Overall, 6778 patients were included in the study. 52 patients died and 628 patients were admitted to hospital. The adjusted mortality risk ratio given a response time of $\geq 8$ minutes was 0.635 (95\% CI: $0.346-1.166 ; p=0.143)$. The adjusted hospital admission risk ratio given a response time of $\geq 8$ minutes was 1.165 (95\% CI: 0.985-1.379; $\mathrm{p}=0.075$ ).

Conclusions A response time of $\geq 8$ minutes was not associated with a difference in all cause mortality or hospital admission for paediatric patients suffering from a traumatic injury.

\section{EPIDEMIOLOGY OF INJURIES LEADING TO VOLUNTARY WITHDRAWAL FROM US HIGH SCHOOL SPORTS, 2005/ 06-2014/15}

'Lauren A Pierpoint, ' Dustin W Currie, ${ }^{2}$ Sarah K Fields, ${ }^{1}$ R Dawn Comstock. ${ }^{1}$ Program for Injury Prevention, Education and Research (PIPER), Department of Epidemiology, Colorado School of Public Health, Aurora, CO, USA; ${ }^{2}$ Department of Communication, University of Colorado Denver, USA

\subsection{6/injuryprev-2016-042156.160}

Background Over 7 million US high school athletes played sports in 2013/14. Participation elevates injury risk, which sometimes results in athletes' voluntarily withdrawing from sports. Understanding what prompt athletes to withdraw, despite no medical disqualification, is important to inform injury prevention and management to reduce dropout rates.

Methods We utilised the High School Reporting Information Online database. Athlete exposure (AE) and injury incidence data were collected from a large national sample of US high schools for 22 sports from 2005/06-2014/15. Injuries motivating voluntary withdrawal from sports participation, despite no medical disqualification, were analysed.

Results Overall, 794 injuries resulting in voluntary withdrawal (representing $1.2 \%$ of all injuries reported) occurred during 35,454,673 AEs (rate $=2.24 / 100,000$ AEs). Boys' wrestling had the highest rate (6.17) followed by football (5.15), girls' track and field (1.96) and girls' soccer (1.68). Concussions accounted for $24.6 \%$ of these injuries, ligament sprains for $22.5 \%$, and muscle strains for $12.2 \%$. In gender-comparable sports, there were no differences in the proportion (\# of withdrawals/\# all injuries) of boys' withdrawals compared to girls' (Injury Proportion Ratio $=1.19 ; 95 \%$ CI: $=0.92-1.53)$. Most injuries were new $(77.8 \%)$ and few required surgery (6.5\%). Trends over time in overall rates of athletes choosing to withdraw were stable, but the rate of withdrawal due to concussion increased significantly (0.12 in 2005/06; 1.01 in 2014/15; p < 0.05).

Conclusions Rates of athletes' voluntary withdrawal, despite no medical disqualification, differed by sport. Concussions were the primary diagnosis; rates of withdrawal due to concussion increased over time. This may reflect increased concussion awareness driving parent or athlete prompted reluctance to return to play. Sport and diagnosis specific targeted injury prevention efforts are needed to reduce voluntary athlete withdrawal from sports.

\section{Injury Monitoring}

Parallel Mon 2.6

\section{AN EVALUATION OF CDC'S WEB-BASED INJURY STATISTICS QUERY AND REPORTING SYSTEM (WISQARS)}

Mick Ballesteros, Sally Thigpen, Mark Faul, Marci-jo Kresnow, Jennifer Middlebrooks. National Centre for Injury Prevention and Control, CDC, USA

\subsection{6/injuryprev-2016-042156.161}

Background WISQARS is an interactive, web-based data query system (WBDQS) that is accessible from the internet. It includes modules for fatal and non-fatal injuries, a separate module on violent deaths, and injury costs and maps. Data come from a variety of trusted sources, including national health surveys and health data repositories. CDC created WISQARS in 1999 to meet the data needs of injury practitioners in the United States. Since that time, the audience has expanded to include researchers, policy makers, media, and the general public.

Objective The purpose of this evaluation was to assess the focus, quality, usefulness, impact, and outcomes of WISQARS; and to identify gaps and areas for improvement. Data were collected through peer-reviewed and grey literature searches, google searches, an environmental scan of internal and external WBDQS, and a series of stakeholder interviews.

Results WISQARS is used as a data source by NGOs, academic institutions, other U.S. federal agencies, and social media websites. Stakeholders most frequently used the fatal and non-fatal modules. The most frequently accessed data were on suicides, poisonings, homicides, motor vehicle crashes, and falls. WISQARS is most often used to respond to data requests, educate decision makers, conduct basic analyses, and teach and plan. Areas for improvement included building more capacity for data visualisations and for users to export both data and graphics, allowing for full mobile responsiveness when accessing, expanding by incrementally including additional data, and developing better support information and guidance on use.

Conclusions While WISQARS has been largely a success in expanding access to U.S. injury and violence surveillance data, there are several opportunities to enhance the functionality of the system for the end user. CDC is planning to use innovations in data science to enhance WISQARS's capacity.

\section{2 IMPLEMENTATION OF A MORTUARY-BASED FATAL INJURY SURVEILLANCE(FIS) SYSTEM IN RURAL AND URBAN HOSPITALS IN TANZANIA (2010-2015)}

${ }^{1}$ Ahmed Makata, ${ }^{1}$ Ruge Manyere, ${ }^{2}$ Charles Massambu, ${ }^{3}$ Amos Mwakigonja, ${ }^{4}$ Kidist Bartolomeous. ${ }^{1}$ Police Forensic Bureau, Tanzania; ${ }^{2}$ MoHSW, Tanzania; ${ }^{3}$ Muhimbili University of Health and Allied Science, Tanzania; ${ }^{4}$ World Health Organisation, Geneva

\subsection{6/injuryprev-2016-042156.162}

Background Tanzanian law states that all injury deaths should undergo a postmortem medical examination. In absence of effective vital registration (VR) systems, mortuaries provide an alternative source of cause of death information to support injury prevention policies and programs. In 2010, a mortuary-based fatal injury surveillance system was established at selected mortuaries and a pilot study commenced with financial and technical support from WHO. 